\title{
Validating the bidimensional reconceptualisation of the autism phenotype
}

\author{
David Skuse ${ }^{1 *}$, William Mandy ${ }^{2}$, Joey Tang ${ }^{3}$
}

\begin{abstract}
We re-evaluated, using dimensional measures, the conventional ICD-10 triadic model of autistic symptomatology in a large clinically heterogeneous population of children with and without autism spectrum disorder (ASD). Conventionally defined elements of the autistic symptom triad were measured in 816 individuals (462 with ASD, 59 with DSM-5 defined Social Communication Disorder and 295 normal or miscellaneous clinical controls). Data output emulated those generated using the ADI-R algorithm. Measured dimensionally, social reciprocity and communication deficits were highly correlated and these symptom scores were therefore combined to create a new variable, namely social-communication impairment which was only moderately correlated with the restricted interests/stereotypy dimension. Multinomial logistic regression revealed a bidimensional model of symptom severity provided the most parsimonious fit to the data and predicted group membership as well as one based on traditional triadic symptomatology. A unidimensional model of autistic symptomatology was comparatively unsatisfactory, failing to predict any cases of Asperger syndrome or pragmatic language disorder. Our findings support the DSM-5 conceptualisation of ASD. Nevertheless, we caution that the boundaries of ASD remain ill-defined and potentially arbitrary.
\end{abstract}

Keywords: autism spectrum disorder, autism phenotype, bidimensional model

\section{INTRODUCTION}

In the 1970s, one of the first epidemiological investigations of autism, among children living in an area of London (1) found disorders of social interaction, communication and imagination occurred together. The severity of these symptoms was highly variable from child to child, associated in some cases with repetitive activities and limited interests. Early infantile autism (2) was not considered to represent a separate category (3). This pioneering study presaged more recent debate that has conceptualised autism as a spectrum of related disorders (4). Studies of conventionally defined symptoms of autism (as measured, for instance, by the Autism Diagnostic Interview - Revised (ADI-R) (5)) indicate impairments in social and communication skills (which are distinct elements within both the International Statistical Classification of Diseases and Related Health Problems, tenth revision (ICD-10) (6) and the Diagnostic and Statistical Manual of Mental Disorders, fourth edition, text revision (DSM-IV TR) (7) classifications) do not load onto separate factors in principal components analysis (8-10). Variants in the domain of restricted interests, whilst not adequately specified in "high functioning" autism (11), may reflect genetically distinct subtypes (12).

Conventional assessments of autism for research purposes (5, 13) have rarely been used to assess autistic symptomatology in typically developing children. The implicit assumption of these "gold standard" instruments, that autistic disorders differ qualitatively from normal development, has been challenged by recent epidemiological findings (14). Until the advent of DSM-5, diagnostic criteria for autism were treated for clinical purposes as categorical, based on three domains of symptomatology that comprised: first, reciprocal social interaction skills; second, communication skills; and third, repetitive or stereotyped behaviours. Children with severe autistic symptoms in two of the three domains but who lacked significant problems in the third - almost always stereotyped behaviours and restricted interests (15) - could not fulfil DSM-IV TR or ICD10 criteria. Now, in the context of DSM-5, there are equivalent specific criteria for making diagnostic decisions in the two domains of impairment, social communication and social interaction, and

${ }^{1}$ Great Ormond Street Institute of Child Health, University College London, London, UK. ${ }^{2}$ Division of Psychology and Language Sciences, University College London, London, UK. ${ }^{3}$ Myndful Learning Association, Hong Kong SAR, China.

*Corresponding author. Email: d.skuse@ucl.ac.uk (D.S.) restricted, repetitive patterns of behaviour, interests or activities.

For many years, diagnostic systems have drawn a distinction between autism and specific language impairment (SLI) (4, 16-18). However, in adulthood, autistic behaviours may be found in former SLI children (19) and, in families with an SLI child, siblings have an increased risk of autistic disorders (20). More blurring of diagnostic boundaries was evidenced by the condition "pragmatic language impairment" (21) and, more recently, by "social (pragmatic) communication disorder" (22) in which a characteristic impairment of language used for social communication is associated with autistic traits in the presence of relatively good social skills (23). Where are the boundaries of the autistic spectrum? Are symptom clusters tridimensional, bidimensional or unidimensional (24)? We investigated these concepts in a heterogeneous population of children comprising autism spectrum disorders, other clinical conditions and typically developing general population samples.

\section{RESULTS}

Testing the three-dimensional model of autistic symptomatology We first tested the fit of the conventional triadic model of autistic symptomatology to the diagnostic classification outlined above. A multinomial logistic regression was conducted on the three symptom dimensions defined by ICD-10 and DSM-IV TR: (i) reciprocal social interaction, (ii) use of language and other social communication skills and (iii) repetitive and stereotyped behaviour, using all five groups of participants, with the normal/clinical group as the reference. The model chi-square (likelihood ratio test) fit well $\left(\right.$ model $\left.X^{2}{ }_{(12)}=1329.98, p<0.001\right)$ and the Pearson goodness-of-fit was also significant $\left(\mathrm{X}^{2}{ }_{(3208)}=26121.26, p<\right.$ 0.001 ; deviance $\mathrm{X}_{(3208)}=1083.15$, n.s.). Deviance reflects error associated with the model and should be non-significant. Likelihood ratio tests revealed that goodness-of-fit to the five diagnostic category model was most reduced when the reciprocal social interaction measure was omitted $\left(\mathrm{X}^{2}{ }_{(4)}=343.32, p<0.001\right)$, less reduced when the repetitive and stereotyped behaviour measure was omitted $\left(\mathrm{X}_{(4)}^{2}=242.43, p<0.001\right)$ and least affected by omitting language and other social communication skills $\left(\mathrm{X}^{2}(4)\right.$ $=64.73, p<0.001)$. Severity of impairment in reciprocal social interaction and use of language and other social communication skills predicted diagnoses of autism, Asperger syndrome, atypical 
autism and pragmatic language impairment. Severity of repetitive and stereotyped behaviour significantly predicted autism, Asperger syndrome and pragmatic language impairment but not atypical autism (Table 1). When all three symptom dimensions were included, the model correctly predicted $60.1 \%$ of the autism cases, $23.9 \%$ of the Asperger syndrome cases, $87.4 \%$ of the atypical autism cases, $16.9 \%$ of the pragmatic language disorder cases and $93.6 \%$ of the normal/clinical cases. Overall correct prediction was $71.4 \%$.

Table 1. Parameter estimates in a multinomial logistic regression with three predictor variables (the triadic model)

\begin{tabular}{|l|l|c|c|}
\hline Group & Symptom severity scale & B & Wald \\
\hline \multirow{4}{*}{ Autism } & (i) reciprocal social interaction & 1.66 & $90.11^{* *}$ \\
& (ii) use of language and other social communication skills & 0.65 & $41.99^{\star *}$ \\
& (iii) repetitive and stereotyped behaviour & 0.75 & $30.08^{* \star}$ \\
\hline \multirow{2}{*}{ Asperger } & (i) reciprocal social interaction & 1.61 & $85.51^{* \star}$ \\
Syndrome & (ii) use of language and other social communication skills & 0.56 & $31.30^{* \star}$ \\
& (iii) repetitive and stereotyped behaviour & 0.79 & $33.61^{* \star}$ \\
\hline \multirow{2}{*}{ Atypical } & (i) reciprocal social interaction & 1.61 & $88.64^{* *}$ \\
Autism & (ii) use of language and other social communication skills & 0.61 & $41.04^{* \star}$ \\
& (iii) repetitive and stereotyped behaviour & -0.22 & 2.63 \\
\hline Social & (i) reciprocal social interaction & 0.30 & $10.17^{\star *}$ \\
Communication & (ii) use of language and other social communication skills & 0.35 & $27.19^{\star *}$ \\
Disorder & (iii) repetitive and stereotyped behaviour & 0.24 & $5.14^{*}$ \\
\hline
\end{tabular}

The B statistic is an estimated logistic coefficient of the constant (also called the 'intersect') for each predictor variable. The Wald chi-square tests the null hypothesis that the constant equals zero. The closer a logistic coefficient is to zero, the less influence the predictor has in predicting the logit, where logit is what is being predicted, i.e. the odds of membership in the category of the outcome variable. The referent population here is the normal/clinical group against which each diagnostic group is estimated with respect to the three symptom dimensions. ${ }^{* *} p<0.001 ;{ }^{*} p<0.050$

\section{Correlations between symptom measures}

Across all diagnostic groups, the reciprocal social interaction dimension and the use of language and other communication skills dimension correlated very highly with one another (Pearson $\mathrm{r}=$ $0.81, p<0.001$; see Fig. 1).

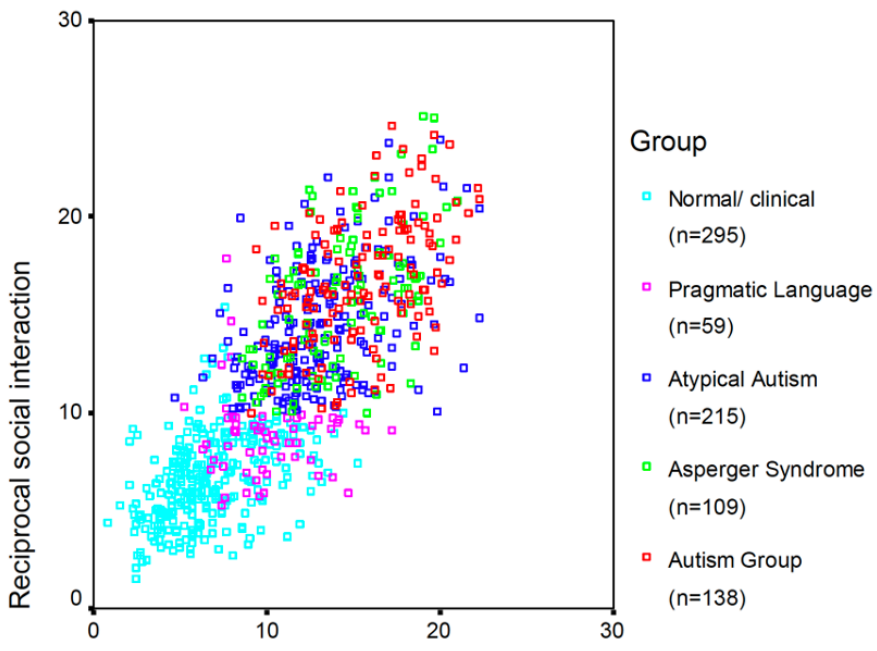

Use of language and other social communication skills

Fig. 1. Scatterplot showing the relationship between (i) reciprocal social interaction and (ii) use of language and other social communication skills.

There is no direct counterpart to $\mathrm{R}^{2}$ in logistic regression, so Variance Inflated Factor (VIF) test for collinearity could not be computed but clearly there is a collinearity problem. Individually, these two dimensions were only moderately correlated with repetitive and stereotyped behaviours (both Pearson $r=0.61, p<0.001$ ). Dimensional scores representing (i) reciprocal social interaction and (ii) use of language and other social communication skills were therefore weighted and combined to form a new variable (i+ii) "social interaction and communication" to which each contributed $50 \%$. The two dimensions (i) and (ii) had different possible maximum scores: 30 and 26 respectively. They were each re-scaled to a possible maximum score of 50 . When combined, the new variable (i+ii) had a possible maximum total of 100 . In this way, the two original variables contributed equally.

\section{Fitting a new bidimensional model of autistic symptomatology}

We then conducted a second multinomial regression using two symptom dimensions as predictors: (i+ii) social interaction and communication and (iii) repetitive and stereotyped behaviour. The chi-square model fit well (model $\left.\mathrm{X}^{2}{ }_{(8)}=1248.53, p<0.001\right)$ and the Pearson goodness-of-fit was significant $\left(\mathrm{X}^{2}{ }_{(3212)}=5313.96, p\right.$ $<0.001$; deviance $\mathrm{X}^{2}{ }_{(3212)}=1164.60$, n.s.). Likelihood ratio tests revealed that fit was more reduced when the social interaction and communication measure was omitted $\left(\mathrm{X}^{2}{ }_{(4)}=676.64, p<0.001\right)$ than the repetitive and stereotyped behaviour measure $\left(\mathrm{X}^{2}{ }_{(4)}=\right.$ $240.42, p<0.001)$. Both dimensions significantly predicted autism, Asperger's syndrome, atypical autism and pragmatic language impairment (Social Communication Disorder) but repetitive and stereotyped behaviour was relatively weaker as a predictor for atypical autism and pragmatic language impairment. Table $\mathbf{2}$ shows the parameter estimates for the different groups. This bidimensional model correctly predicted $61.6 \%$ of the autism cases, $23.9 \%$ of the Asperger syndrome cases, $84.2 \%$ of the atypical autism cases, $3.4 \%$ of the pragmatic language disorder cases and $93.2 \%$ of the normal/clinical cases. Overall correct prediction was $69.7 \%$, little different from the triadic model $(71.4 \%)$.

Table 2. Parameter estimates in a multinomial logistic regression with two predictor variables (a new bidimensional model).

\begin{tabular}{|l|l|c|c|}
\hline Group & Symptom severity scale & B & Wald \\
\hline \multirow{2}{*}{ Autism } & (i+ii) social interaction and communication & 0.51 & $137.16^{\star \star}$ \\
& (iii) repetitive and stereotyped behaviour & 0.70 & $30.49^{* \star}$ \\
\hline \multirow{2}{*}{ Asperger Syndrome } & (i+ii) social interaction and communication & 0.48 & $118.83^{\star \star}$ \\
& (iii) repetitive and stereotyped behaviour & 0.74 & $34.27^{* \star}$ \\
\hline \multirow{2}{*}{ Atypical Autism } & (i+ii) social interaction and communication & 0.49 & $131.53^{\star \star}$ \\
& (iii) repetitive and stereotyped behaviour & -0.26 & $4.44^{\star}$ \\
\hline Social Communication & (i+ii) social interaction and communication & 0.18 & $43.69^{\star \star}$ \\
Disorder & (iii) repetitive and stereotyped behaviour & 0.25 & $5.42^{\star}$ \\
\hline
\end{tabular}

The B statistic is an estimated logistic coefficient of the constant (also called the 'intersect') for each predictor variable. The Wald chi-square tests the null hypothesis that the constant equals zero. The closer a logistic coefficient is to zero, the less influence the predictor has in predicting the logit, where logit is what is being predicted, i.e. the odds of membership in the category of the outcome variable. The referent population here is the normal/clinical group against which each diagnostic group is estimated with respect to the two symptom dimensions. ${ }^{* *} p<0.001 ;{ }^{*} p<0.050$

We also examined the fit of a unidimensional model to the data, using a single variable created by combining the three original dimensions with equal weighting. The three dimensions (i), (ii) and (iii) had different possible maximum scores: 30, 26 and 12 respectively. They were each re-scaled to a possible maximum score of 33.33. When combined, the new variable had a possible maximum total of 100 . In this way, the three original variables contributed equally. This model was substantially less satisfactory in terms of diagnostic prediction. The chi-square model fit well (model $\left.\mathrm{X}_{(4)}^{2}=1017.14, p<0.001\right)$ and the Pearson goodness-of- 
fit was significant $\left(\mathrm{X}^{2}{ }_{(3216)}=4062.24, p<0.001\right.$; deviance ${ }^{2}{ }_{(3216)}$ $=1395.99$, n.s.). However, overall, only $64.3 \%$ of individuals were correctly classified and this included no case of Asperger syndrome or pragmatic language disorder $(71 \%$ autism, $74.4 \%$ atypical autism and $90.5 \%$ normal/clinical cases).

\section{Correlations between symptom dimensions in the bidimensional model}

When all five groups (including the normal/clinical group) were considered together, the two measures contributing to the bidimensional model (i+ii and iii) were moderately correlated (Pearson $\mathrm{r}=$ $0.64, p<0.001$; see Fig. 2). Fig. 3 and 4 give the distribution of these symptom dimensions. The combined social interaction and communication scores had an approximately normal distribution across the entire sample: (skewness $=0.04$, standard error $=0.08$ ) (Fig. 3). In contrast, the repetitive and stereotyped behaviour scores were positively skew (skewness $=1.02$, standard error $=0.08$ ), reflecting a floor effect (Fig. 4).

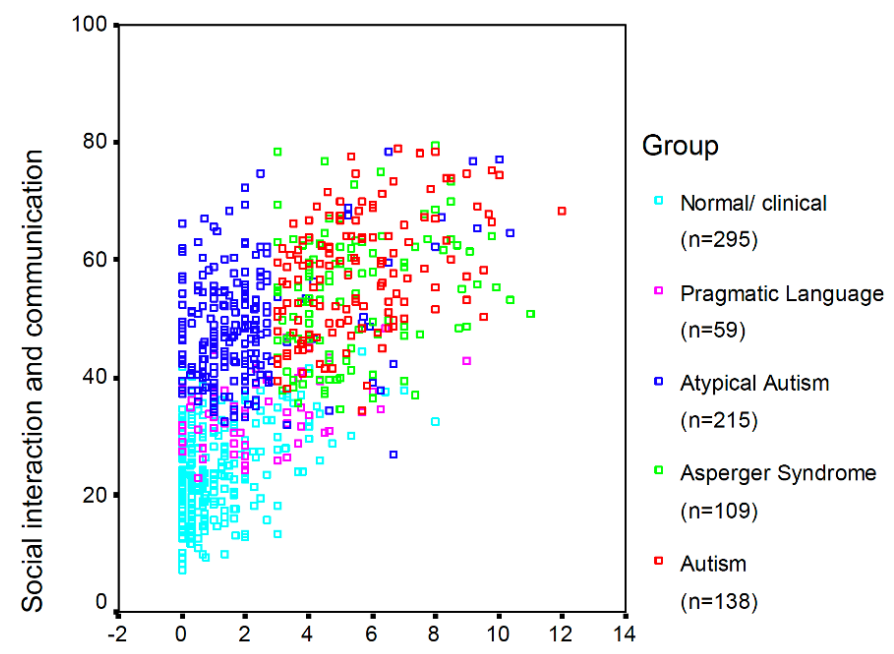

\section{Repetitive and stereotyped behaviour}

Fig. 2. Scatterplot showing the relationship between $(i+i i)$ social interaction and communication and (iii) repetitive and stereotyped behaviour.

\section{Correlations between symptom dimensions and IQ}

Partial correlations (controlling for age and gender) were calculated between IQ scores and symptom severity measures for the two dimensions: (i+ii) social interaction and communication, and (iii) repetitive and stereotyped behaviour. They were in the range 0.01 to 0.09 and all failed to reach significance.

\section{Bidimensional model as a predictor of pervasive developmental disorder (PDD) diagnostic categories}

We tested the hypothesis that the conventionally specified diagnostic categories of autism simply reflect differences in severity of symptomatology, measured dimensionally. A multivariate ANOVA was performed on the two symptom dimension measures: (i+ii) social interaction and communication, and (iii) repetitive and stereotyped behaviour. It revealed a significant main effect of group for both measures $\left(\mathrm{F}_{(4,811)}=514.25, p<0.001\right.$ and $\mathrm{F}_{(4,811)}=$ $244.69, p<0.001$ respectively). Table 3 shows the mean differences across groups on these measures. Tukey HSD tests were used to test for significant differences. The three ASD groups and the pragmatic language disorder (Social Communication Disorder) group had significantly more severe symptomatology than the normal/clinical group (all $p<0.001$ ).

Table 3. Mean differences between diagnostic groups on the bidimensional symptom severity scales: (i+ii) social interaction and communication and (iii) repetitive and stereotyped behaviour.

\begin{tabular}{|c|c|c|c|}
\hline \multirow[b]{2}{*}{ Group (I) } & \multirow[b]{2}{*}{ Group (J) } & \multicolumn{2}{|c|}{ Mean Difference (I-J) } \\
\hline & & $\begin{array}{c}\text { (i+ii) Social } \\
\text { interaction and } \\
\text { communication }\end{array}$ & $\begin{array}{l}\text { (iii) Repetitive and } \\
\text { stereotyped } \\
\text { behaviour }\end{array}$ \\
\hline \begin{tabular}{|l} 
Autism \\
(i+ii) mean $=57.60$ \\
(iii) mean $=5.50$
\end{tabular} & $\begin{array}{l}\text { Asperger Syndrome } \\
\text { Atypical Autism } \\
\text { Social Communication } \\
\text { Normal/ Clinical }\end{array}$ & $\begin{array}{c}3.90^{*} \\
8.54^{\star *} \\
23.26^{\star *} \\
34.09^{\star \star}\end{array}$ & $\begin{array}{c}0.15 \\
3.59^{\star *} \\
3.12^{\star *} \\
4.48^{\star *}\end{array}$ \\
\hline $\begin{array}{l}\text { Asperger Syndrome } \\
\text { (i+ii) mean }=53.70 \\
\text { (iii) mean }=5.36\end{array}$ & $\begin{array}{l}\text { Autism } \\
\text { Atypical Autism } \\
\text { Social Communication } \\
\text { Normal/ Clinical }\end{array}$ & $\begin{array}{c}-3.90^{\star} \\
4.64^{\star \star} \\
19.36^{\star \star} \\
30.19^{\star \star}\end{array}$ & $\begin{array}{l}-0.15 \\
3.44^{\star \star} \\
2.97^{\star \star} \\
4.33^{\star \star}\end{array}$ \\
\hline $\begin{array}{l}\text { Atypical Autism } \\
\text { (i+ii) mean }=49.06 \\
\text { (iii) mean }=1.91\end{array}$ & $\begin{array}{l}\text { Autism } \\
\text { Asperger Syndrome } \\
\text { Social communication } \\
\text { Normal/ Clinical }\end{array}$ & $\begin{array}{l}-8.54^{* *} \\
-4.64^{* *} \\
14.72^{* *} \\
25.55^{\star *}\end{array}$ & $\begin{array}{c}-3.59^{* *} \\
-3.44^{* *} \\
-0.47 \\
0.89^{* *}\end{array}$ \\
\hline $\begin{array}{l}\text { Social Communication } \\
\text { Disorder } \\
\text { (i+ii) } \text { mean }=34.34 \\
\text { (iii) mean }=2.38\end{array}$ & $\begin{array}{l}\text { Autism } \\
\text { Asperger Syndrome } \\
\text { Atypical Autism } \\
\text { Normal/ Clinical }\end{array}$ & $\begin{array}{l}-23.26^{\star \star} \\
-19.36^{\star \star} \\
-14.72^{\star \star} \\
10.83^{\star \star}\end{array}$ & $\begin{array}{c}-3.12^{\star \star} \\
-2.97^{\star \star} \\
0.47 \\
1.36^{\star \star}\end{array}$ \\
\hline $\begin{array}{l}\text { Normal/ Clinical } \\
\text { (i+ii) mean }=23.51 \\
\text { (iii) mean }=1.02\end{array}$ & $\begin{array}{l}\text { Autism } \\
\text { Asperger Syndrome } \\
\text { Atypical Autism } \\
\text { Social Communication }\end{array}$ & $\begin{array}{l}-34.09^{\star \star} \\
-30.19^{\star \star} \\
-25.55^{\star \star} \\
-10.83^{\star \star}\end{array}$ & $\begin{array}{l}-4.48^{\star \star} \\
-4.33^{\star \star} \\
-0.89^{\star \star} \\
-1.36^{\star *}\end{array}$ \\
\hline
\end{tabular}

On the social interaction and communication measure, significant differences were observed between all the groups - the autism sample was most impaired, scoring significantly higher than the Asperger syndrome group $(p<0.050)$, which in turn scored significantly higher than the atypical autism group $(p<0.001)$. The subgroup with Social Communication Disorder was significantly less impaired on this measure than all three ASD groups (all $p$ $<0.001)$ but significantly more impaired than the normal/clinical group $(p<0.001)$.

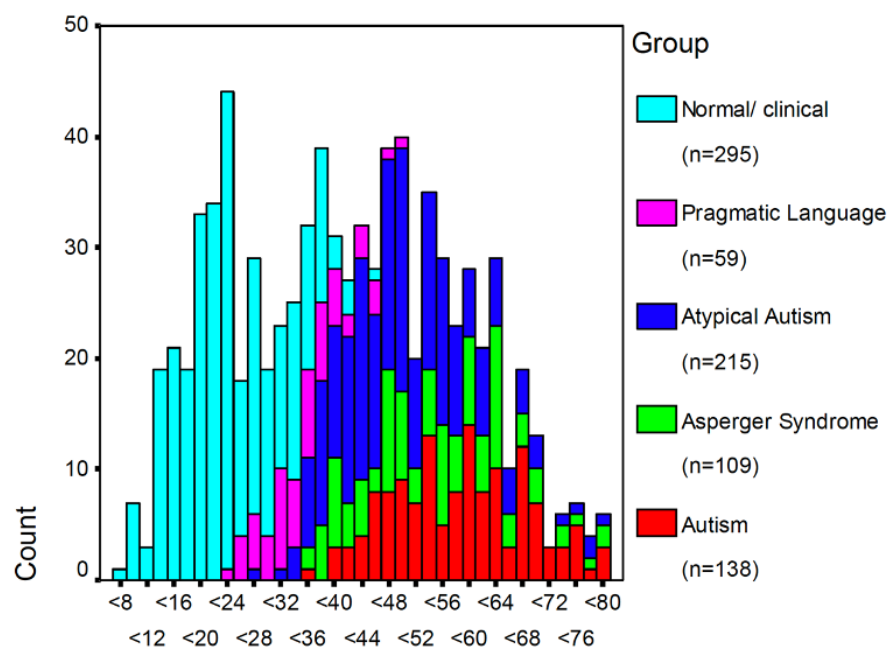

Social interaction and communication

Fig. 3. Histogram showing distribution of social interaction and communication scores.

On the repetitive and stereotyped behaviour measure, the autism and the Asperger syndrome groups were not significantly differentiated but both scored higher than the children with atypical autism $(p$ 
$<0.001)$. The latter were not differentiated on this measure from the Social (Pragmatic) Communication Disorder group but both scored significantly higher than the normal/clinical group $(p<0.001)$.

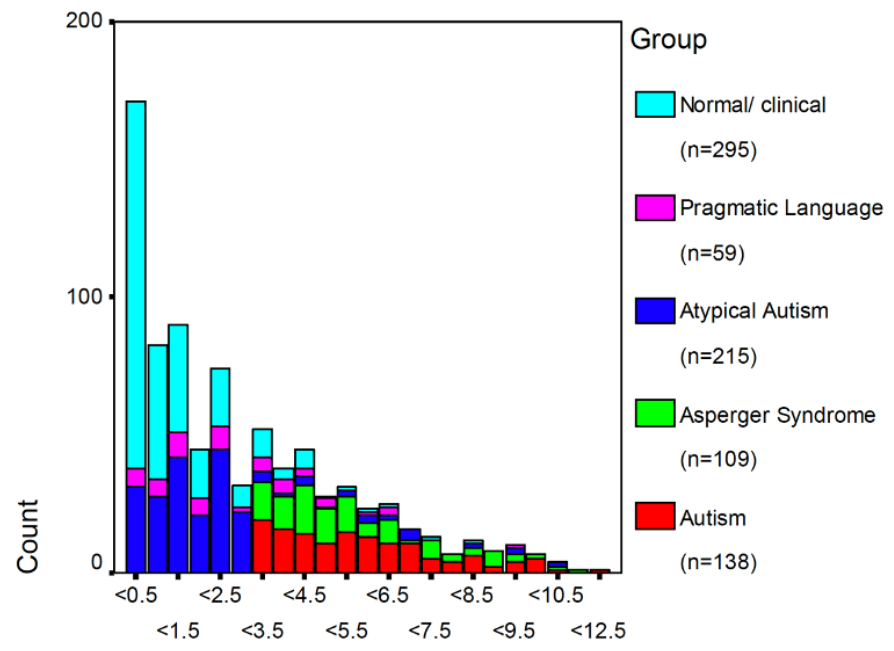

Repetitive and stereotyped behaviour

Fig. 4. Histogram showing distribution of repetitive and stereotyped behaviour scores.

\section{DISCUSSION}

We have reassessed the traditional (DSM-IV TR/ICD-10) triad of impairment used to define autism spectrum disorders. Social reciprocity, pragmatic language impairments and repetitive behaviours were examined quantitatively in a large heterogeneous population of children who were mostly of normal-range intelligence, including $26.2 \%$ with no clinical diagnosis. We found the social and communication elements of the triad are closely correlated across the full range of clinical severity. The distribution of severity scores in the combined social interaction and communication variable (Fig. 3) approximates statistical normality. In contrast, stereotyped and repetitive behaviours are less closely correlated with both of the other elements of the triad, and the distribution of severity is truncated at the lower (less severe) end (Fig. 4), implying the instrument used to identify such behaviours is relatively insensitive and should be improved. The high correlation between social and language deficits is consistent with previous research on smaller, more clinically homogeneous, samples. Our findings support the decision made to revise the definition of autism in DSM-5 (15) and the associated reconceptualisation of the autism phenotype for both clinical and research purposes, which has been recapitulated in ICD-11.

We used the symptom severity measures, based on the ADI$\mathrm{R}$ algorithm, to predict membership of five mutually exclusive diagnostic groups, which had been assigned from interview data using DSM-IV/ICD-10 criteria. Using multinomial logistic regression to model the data, a parsimonious bidimensional model predicted group membership as well as one based on triadic symptomatology. Both enabled us to classify approximately $70 \%$ of all the participants of our investigation into mutually exclusive diagnostic categories. We found evidence that DSM-IV/ICD-10 specified categories of autistic disorder do not reflect conditions with distinctive aetiologies but that the analysis pointed to the validity of the DSM-5 revision (25). Whilst it was possible to apportion study participants into four conventionally labelled diagnostic categories (autism, Asperger syndrome, atypical autism, Social Communication Disorder, plus a fifth comprising normal/clinical group) using data from the ADI-R algorithm (plus additional DSMIV TR criteria), they are essentially on a continuum of severity in terms of social and communication skills (26). There is no clear boundary between the normal/clinical group and those with "atypical autism". If we ignore diagnosis and assume that we had simply measured the severity of autistic symptomatology on two dimensions, inspection of Fig. 1-4 would tell us nothing useful about where to draw the line between normality and clinical impairment. Whilst the data do not support the theory that the spectrum of autistic symptomatology is unidimensional (10), the floor-effect associated with the distribution of stereotyped and repetitive behaviours (contrast Fig. 3 and 4) reduced this variable's potential correlation with the other elements of the triad. A major effort would be required to refine and increase the sensitivity of measurement of stereotyped behaviours and restricted interests in order to investigate this matter adequately.

Our main objective was to investigate the boundaries of the autism spectrum disorders using dimensional scores based upon interview-based symptomatology. The diagnoses we derived do not necessarily correspond to the clinical diagnoses that would have been assigned if all available data (e.g. the Autism Diagnostic Observation Schedule, school observation, etc.) had been taken into consideration. Further work would be needed to discover if other autism subcategories: i) comprise distinct symptom clusters within the domain of content used by the diagnostic algorithms; ii) are differentiable in terms of associated symptoms that do not enter the diagnostic algorithms (e.g. sleep patterns, regression, motor skills, etc.); iii) are associated with markedly different degrees of symptomatology (excluding algorithm components) in terms of current or future adjustments and life skills. The purpose of the analyses presented here is to show the inherent dimensionality of autistic symptoms, using an internally coherent and consistent set of measures that could be applied to all participants equally, whether they had been clinically referred or not. Clinical diagnosis, in contrast, is necessarily subjective and comprises the evaluation and synthesis of often conflicting evidence (23). It is not possible from this study to draw any conclusions about where exactly the clinical boundaries between autism spectrum disorders and neurotypicality should be placed. Independent evaluation of functional impairment in everyday life would be required to begin to address that critical question but would inevitably run into issues of undue complexity. For instance, should the boundaries vary by age or by gender? For now, we must accept the distinction between neurotypicality and clinical significance is subject to an arbitrary consensus, which has no external validity, for instance, in terms of genetic risk (27).

Our study was one of the first to incorporate detailed symptomatic analysis of autism in a large sample without mental retardation. Measures of IQ and autistic symptomatology are not tightly correlated according to our findings. By far the largest proportion of children being diagnosed nowadays have normalrange IQ (28). In the general population, high verbal IQ tends to protect females from exhibiting autistic traits yet increases the risk for male children (29). We may have failed to find a relationship between IQ and symptom severity because there were few children in the sample as a whole with very low non-verbal IQ $(9.1 \%<70)$. No child in the sample had a non-verbal IQ $<50$ and few $(3.1 \%)$ had no spoken language, hence averting the potentially confounding effect of developmental delay, which can lead to serious difficulties in the evaluation of clinical validity of autistic symptoms (30). 
There has been recent debate concerning whether DSM-5 defined Social (Pragmatic) Communication Disorder (SPCD) is simply another term for a disorder on the autistic spectrum (23). Our SPCD sample was impaired in terms of communication skills compared to the normal/clinical group but their reciprocal social interaction abilities were good in contrast to children with ASD. However, many also had significantly more autistic-like repetitive and stereotyped behaviours than normal/clinical samples, highlighting the difficulties inherent in distinguishing the autism spectrum from pragmatic language disorders (21).

In summary, our findings confirm earlier research indicating autistic symptomatology is dimensional rather than qualitatively distinct from normal-range behaviour. Two dimensions, reciprocal social/communication impairment and repetitive behaviours/restricted interests parsimoniously model the clinical diversity of autism spectrum disorders. Conventionally specified nosological categories of diagnosis can be predicted from bidimensional symptom severity scores. Nevertheless, we have shown clearly that the boundaries of the autistic spectrum are not clearly defined and the validity of diagnostic distinctions within that spectrum remains an open question $(31,32)$.

\section{MATERIALS AND METHODS}

\section{Developmental, Dimensional and Diagnostic Interview}

The Developmental, Dimensional and Diagnostic Interview (3di) provides an efficient and accurate means of assessing, in dimensional terms, the presence of autistic symptoms in both clinical and normal populations (33). It comprises a computerised procedure, for administration by trained interviewers, which generates symptomatic and diagnostic profiles for both autism and non-autistic conditions. Test-retest and inter-rater reliabilities are excellent as is criterion validity, a comparison with the ADI-R, from which it differs mainly in terms of the detail of the clinical annotation used for algorithm generation. This is considerably greater in the 3di, hence allowing much finer discriminations to be made within the clinical spectrum of disorders. The interview was devised from the outset to be appropriate for administration to parents of nonsymptomatic children. Each individual response can be downloaded for statistical analysis. For the purpose of this paper, we used the option offered by the $3 \mathrm{di}$ to emulate the dimensions and the symptom severity scores calculated by the ADI-R algorithm (34).

\section{Interviewers}

Interviewers for the study consisted of seventeen psychiatrists, four clinical psychologists, four paediatric neurologists and one community paediatrician. All interviewers were trained on the assessments of the instrument. Assessments of heterogeneous clinical populations were conducted in London, Oxford, Sunderland and Luton, UK and in Tampere, Finland, the latter using a Finnish translation of the $3 \mathrm{di}$.

\section{Children's Communication Checklist (CCC)}

The Children's Communication Checklist (CCC) was developed to discriminate between children with a purely pragmatic language disorder and children on the autistic spectrum (35). It was originally devised for use by teachers but has been shown to give valid ratings when completed by parents (36). We incorporated the original version of this instrument in the 3di, for administration either as an interview or (pre-rated) questionnaire. Scoring procedures were identical to those used in previous research (35). We used this instrument to emulate the diagnostic criteria for DSM-5 defined
Social Communication Disorder.

\section{Assessment of cognitive abilities}

Performance IQ was assessed using the Ravens Coloured Progressive Matrices (37) whilst an estimate of verbal IQ was obtained using the British Picture Vocabulary Scale (38). Other assessments included the Wechsler Abbreviated Scale of Intelligence (39) and the Wechsler Intelligence Scale for Children (40). IQ was only assessed in the British sample.

\section{Participants}

Children were recruited into this study from a variety of sources. First, we took consecutive referrals to pervasive developmental disorder services within specialist centres in London, UK and Tampere, Finland. Children with severe learning difficulties or who had no spoken language were excluded. Second, we took consecutive referrals, with miscellaneous psychiatric problems, to community child mental health clinics in Sunderland and Luton, UK. Third, children were recruited from schools and special classes for children with communication difficulties. Finally, typically developing children were recruited from the general population and from (non-behavioural) paediatric outpatient clinics. Children less than 3 years of age have been excluded from the analyses.

The diagnostic process was based entirely on information obtained from parental interview and teacher report. Information from the 3di enabled classification according to ICD-10 (and DSM-IV TR) criteria for childhood autism, Asperger syndrome, atypical autism (ICD-10) or pervasive developmental disorder not otherwise specified (PDD-NOS) (DSM-IV TR), pragmatic language disorder, and normal or other clinical diagnosis. Diagnostic categories were defined to be mutually exclusive. A diagnosis of autism $(n=138)$ demanded that the child had significant language delay (phrase speech after 36 months) together with scores over threshold according to the ADI-R algorithm. The ADI-R does not give guidance for a diagnosis of Asperger syndrome. Because strictly applying either ICD-10 (or DSM-IV) criteria renders the diagnosis almost impossible in practice, an observation that led to the dropping of the category from DSM$5(41,42)$, we adapted previously established guidelines $(43)$. These require criteria for autism to be met but there must be normal early language development, phrase speech by 36 months and no marked or persistent deviant language development (no delayed echolalia, pronominal reversal or neologisms).

Atypical autism (ICD-10) and PDD-NOS (DSM-IV TR) demanded reciprocal social interaction skill impairment (according to ADI-R algorithm criteria) plus either communication deficits or stereotyped behaviours $(\mathrm{n}=196)$. Most children in these categories $(96.9 \%)$ had social reciprocity and communication scale scores, calculated using the ADI-R algorithm, in the clinical range. The remainder $(3.1 \%)$ met criteria on the social reciprocity and the restricted interests and motor stereotypy scales only. In this category were 19 children who met ADI-R criteria for reciprocal social interaction, communication and stereotyped behaviour but who could not with confidence be assigned to the more specific categories. In 9 cases, there was insufficient information to determine whether language had been delayed. In 10 other instances, despite no language delay (thereby excluding autism), there was severe and persistent language deviance (delayed echolalia, pronominal reversal and neologisms), thereby excluding Asperger syndrome too.

The category of Social (Pragmatic) Communication Disorder 
Table 4. Summary of participant characteristics with symptom severity scores measured using the Autism Diagnostic Interview (ADI-R) algorithm and the Children's Communication Checklist (CCC).

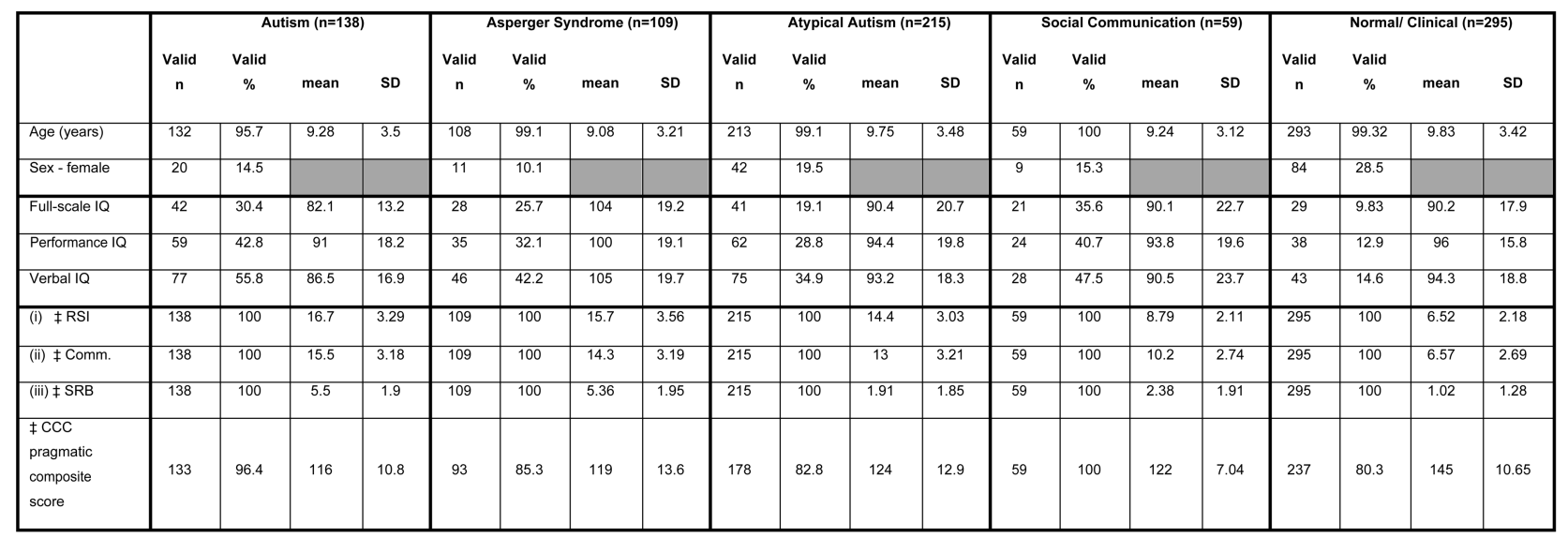

¥Reciprocal social interaction (RSI), communication and language (Comm.) and stereotyped and repetitive behaviours (SRB) were measured using the Autism Diagnostic Interview (ADI-R) algorithm. Pragmatic language score was obtained using the Children's Communication Checklist (CCC).

( $\mathrm{n}=59$ ) comprised a subgroup of those who did not meet criteria for autism, Asperger syndrome or atypical autism. None had any significant structural language impairment. All scored within the range of probable clinical significance (total $<132$ ) on parent- and/or teacher-completed Children's Communication Checklist (35) and a speech and language therapist indicated this was an appropriate diagnosis. A substantial proportion (72.9\%) obtained scores over the threshold for clinical significance on the communication subscale of the ADI-R algorithm; a minority had significant impairment in reciprocal social interaction $(10.2 \%)$ or stereotyped and repetitive behaviours/restricted interests (35.6\%).

Finally, we created a category of typically developing children or who had another clinical diagnosis $(n=295)$, selected from general population samples that had been assessed in the validation of the 3di (24) or from clinical samples of children with nonautism spectrum disorders. According to ICD-10 criteria, $72.6 \%$ of this sample had no psychiatric disorder, $9.7 \%$ had a diagnosis of oppositional defiant disorder (ODD), 6.8\% had a diagnosis of conduct disorder, $6.3 \%$ had a diagnosis of attention deficit hyperactivity disorder (ADHD), 3.4\% had a diagnosis of an anxiety disorder, $0.8 \%$ had a diagnosis of a mood disorder and $0.4 \%$ had a diagnosis of post-traumatic stress disorder (PTSD). Table 4 gives participant details.

\section{ETHICAL PERMISSION}

After complete description of the study to the participants, written informed consent was obtained from both cases and comparisons. The investigation was conducted in accordance with guidelines from the appropriate hospital ethics committees.

\section{REFERENCES}

1. L. Wing, J. Gould, Severe impairments of social interaction and associated abnormalities in children: Epidemiology and classification. J. Autism Dev. Disord. 9, 11-29 (1979).

2. L. Kanner, Autistic disturbances of affective contact. Nerv. Child 2, 217-250 (1943)

3. L. Wing, The continuum of autistic disorders in E. Schopler, G. M. Mesibov(eds), Diagnosis and Assessment in Autism. New York, Plenum, 1988, pp. 91-110.

4. R. Grzadzinski, M. Huerta, C. Lord, DSM-5 and autism spectrum disorders (ASDs): An opportunity for identifying ASD subtypes. Mol. Autism 4, 12 (2013).

5. A. L. Couteur, C. Lord, M. Rutter, The Autism Diagnostic Interview - Revised (ADI- R) (Western Psychological Services, Los Angeles CA, USA, 2003).

6. World Health Organisation, The ICD-10 classification of mental and behavioral disorders: Diagnostic criteria for research (1993).

7. American Psychiatric Association, Diagnostic and Statistical Manual of Mental Disorders,
Fourth Edition, Text Revision (DSM-IV TR) (2000).

8. A. Ronald, F. Happé, P. Bolton, L. M. Butcher, T. S. Price, S. Wheelwright, S. Baron-Cohen, R. Plomin, Genetic heterogeneity between the three components of the autism spectrum: A twin study. J. Am. Acad. Child Adolesc. Psychiatry 45, 691-699 (2006).

9. O. Tadevosyan-leyfer, M. Dowd, R. Mankoski, B. Winklosky, S. Putnam, L. McGrath, H. Tagerflusberg, S. E. Folstein, A principal components analysis of the autism diagnostic interviewrevised. J. Am. Acad. Child Adolesc. Psychiatry 42, 864-872 (2003).

10. J. N. Constantino, R. D. Todd, Autistic traits in the general population: A twin study. Arch. Gen Psychiatry 60, 524 (2003).

11. C. de Giambattista, P. Ventura, P. Trerotoli, M. Margari, R. Palumbi, L. Margari, Subtyping the Autism Spectrum Disorder: Comparison of Children with High Functioning Autism and Asperger Syndrome. J. Autism Dev. Disord. 49, 138-150 (2019).

12. T. Bourgeron, Current knowledge on the genetics of autism and propositions for future research. CR Biol. 339, 300-307 (2016).

13. C. Lord, S. Risi, L. Lambrecht, E. H. Cook, B. L. Leventhal, P. C. Dilavore, A. Pickles, M. Rutter, The autism diagnostic observation schedule-generic: A standard measure of social and communication deficits associated with the spectrum of autism. J. Autism Dev. Disord. 30, 205-223 (2000).

14. E. B. Robinson, , B. S. Pourcain, V. Anttila, J. A. Kosmicki, B. Bulik-Sullivan, J. Grove, J. Maller, K. E. Samocha, S. J. Sanders, S. Ripke, J. Martin, M. V. Hollegaard, T. Werge, D. M. Hougaard, B. M. Neale, D. M. Evans, D. Skuse, P. B. Mortensen, A. D. Børglum, A. Ronald, G. D. Smith, M. J. Daly, Genetic risk for autism spectrum disorders and neuropsychiatric variation in the general population. Nat. Genet. 48, 552-555 (2016).

15. W. Mandy, T. Charman, J. Gilmour, D. Skuse, Toward specifying pervasive developmental disorder-not otherwise specified. Autism Res. 4, 121-131 (2011).

16. J. Gibson, C. Adams, E. Lockton, J. Green, Social communication disorder outside autism? A diagnostic classification approach to delineating pragmatic language impairment, high functioning autism and specific language impairment. J. Child Psychol. Psychiatry 54, 11861197 (2013).

17. T. A. Bennett, P. Szatmari, K. Georgiades, S. Hanna, M. Janus, S. Georgiades, E. Duku, S. Bryson, E. Fombonne, I. M. Smith, P. Mirenda, J. Volden, C. Waddell, W. Roberts, T. Vaillancourt, L. Zwaigenbaum, M. Elsabbagh, A. Thompson, The Pathways in ASD Study Team, Language impairment and early social competence in preschoolers with autism spectrum disorders: A comparison of DSM-5 profiles. J. Autism Dev. Disord. 44, 2797-2808 (2014).

18. S. Reilly, B. Tomblin, J. Law, C. McKean, F. K. Mensah, A. Morgan, S. Goldfeld, J. M. Nicholson, M. Wake, Specific language impairment: A convenient label for whom? Int. J. Lang. Commun. Disord. 49, 416-451 (2014).

19. A. J. O. Whitehouse, H. J. Watt, E. A. Line, D. V. M. Bishop, Adult psychosocial outcomes of children with specific language impairment, pragmatic language impairment and autism. Int. J. Lang. Commun. Disord. 44, 511-528 (2009).

20. N. Marrus, L. P. Hall, S. J. Paterson, J. T. Elison, J. J. Wolff, M. R. Swanson, J. Parish-Morris, A. T. Eggebrecht, J. R. Pruett, H. C. Hazlett, L. Zwaigenbaum, S. Dager, A. M. Estes, R. T. Schultz, K. N. Botteron, J. Piven, J. N. Constantino, IBIS Network, Language delay aggregates in toddler siblings of children with autism spectrum disorder. J. Neurodev. Disord. 10, 29 (2018).

21. D. V. Bishop, Speech and Language Impairments in Children, and others, ed. (Psychology Press, 2014), pp. 113-128

22. L. B. Swineford, A. Thurm, G. Baird, A. M. Wetherby, S. Swedo, Social (pragmatic) communication disorder: A research review of this new DSM-5 diagnostic category. J. Neurodev. Disord. 6,41 (2014)

23. W. Mandy, A. Wang, I. Lee, D. Skuse, Evaluating social (pragmatic) communication disorder J. Child Psychol. Psychiatry 58, 1166-1175 (2017)

24. O. J. Veatch, J. Veenstra-VanderWeele, M. Potter, M. A. Pericak-Vance, J. L. Haines, Genetically meaningful phenotypic subgroups in autism spectrum disorders. Genes Brain Behav. 13, 276285 (2014). 
25. W. P. Mandy, T. Charman, D. H. Skuse, Testing the construct validity of proposed criteria for DSM-5 autism spectrum disorder. J. Am. Acad. Child Adolesc. Psychiatry 51, 41-50 (2012).

26. S. Bölte, E. Westerwald, M. Holtmann, C. Freitag, F. Poustka, Autistic traits and autism spectrum disorders: The clinical validity of two measures presuming a continuum of social communication skills. J. Autism Dev. Disord. 41, 66-72 (2011).

27. E. B. Robinson, B. S. Pourcain, V. Anttila, J. A. Kosmicki, B. Bulik-Sullivan, J. Grove, J. Maller, K. E. Samocha, S. J. Sanders, S. Ripke, J. Martin, M. V. Hollegaard, T. Werge, D. M. Hougaard, B. M. Neale, D. M. Evans, D. Skuse, P. B. Mortensen, A. D. Børglum, A. Ronald, G. D. Smith M. J. Daly, Genetic risk for autism spectrum disorders and neuropsychiatric variation in the general population. Nat. Genet. 48, 552-555 (2016)

28. C. Lord, M. Elsabbagh, G. Baird, J. Veenstra-Vanderweele, Autism spectrum disorder. Lance 392, 508-520 (2018).

29. D. H. Skuse, W. Mandy, C. Steer, L. L. Miller, R. Goodman, K. Lawrence, A. Emond, J. Golding, Social communication competence and functional adaptation in a general population of children: Preliminary evidence for sex-by-verbal IQ differential risk. J. Am. Acad. Child Adolesc. Psychiatry 48, 128-137 (2009).

30. D. H. Skuse, Rethinking the nature of genetic vulnerability to autistic spectrum disorders Trends Genet. 23, 387-395 (2007).

31. J. P. Baker, Autism at 70-Redrawing the boundaries. N. Engl. J. Med. 369, 1089 (2013).

32. H. A. Stessman, R. Bernier, E. E. Eichler, A genotype-first approach to defining the subtypes of a complex disease. Cell 156, 872-877 (2014)

33. D. Skuse, R. Warrington, D. Bishop, U. Chowdhury, J. Lau, W. Mandy, M. Place, The developmental, dimensional and diagnostic interview (3di): A novel computerized assessment for autism spectrum disorders. J. Am. Acad. Child Adolesc. Psychiatry 43, 548-558 (2004).

34. C. Lord, A. Pickles, J. Mclennan, M. Rutter, J. Bregman, S. Folstein, E. Fombonne, M. Leboyer, N. Minshew, Diagnosing autism: Analyses of data from the autism diagnostic interview. J. Autism Dev. Disord. 27, 501-517 (1997).

35. D. V. Bishop, Development of the children's communication checklist (CCC): A method for assessing qualitative aspects of communicative impairment in children. J. Child Psychol. Psychiatry 39, 879-891 (1998).

36. D. V. Bishop, G. Baird, Parent and teacher report of pragmatic aspects of communication: Use of the children's communication checklist in a clinical setting. Dev. Med. Child Neurol. 43, 809
(2001)

37. J. C. Raven, J. H. Court, J. C. Raven, Raven's Progressive Matrices and Vocabulary Scales (NFERNELSON, 1998).

38. L. M. Dunn, The British Picture Vocabulary Scale, Second Edition. (NFER- NELSON, 1997)

39. D. Wechsler, Wechsler Abbreviated Scale of Intelligence (WASI) (The Psychological Corporation. Harcourt Brace \& Company, San Antonio, 1999).

40. C. Woolger, Wechsler Intelligence Scale for Children - Third Edition (WISC-III) (The Psychological Corporation. Harcourt Brace \& Company, San Antonio, 1997).

41. P. A. Tryon, S. D. Mayes, R. L. Rhodes, M. Waldo, Can Asperger's disorder be differentiated from autism using DSM-IV criteria? Focus Autism Dev. Disabil. 21, 2-6 (2006).

42. M. C. Lai, M. V. Lombardo, B. Chakrabarti, S. Baron-Cohen, Subgrouping the autism "spectrum": Reflections on DSM-5. PLoS Biol. 11, e1001544 (2013).

43. P. Szatmari, S. E. Bryson, D. L. Streiner, F. Wilson, L. Archer, C. Ryerse, Two-year outcome of preschool children with autism or Asperger syndrome. Am. J. Psychiatry 157, 1980-1987 (2000)

Acknowledgments: The authors gratefully acknowledge the support of: Margaret Dimmock and Jessica Hulsmeier, Sunderland Royal Hospital; Ms Courtenay Norbury, University of Oxford Prof Mary Robertson and Dr Katherine White; University College London; Prof Tony Charman Institute of Psychiatry, Psychology and Neuroscience; Dr Uttom Chowdhury and Dr Maurice Place, Northumbria University; Mr Richard Warrington and Prof Dorothy Bishop, University of Oxford. We thank the clinicians who assisted with data collection and the families who participated. Funding: This study was supported by grants from the Great Ormond Street Hospital for Children NHS Trust, London and the National Alliance for Autism Research (USA), awarded to Prof Skuse and from the City Hospitals Sunderland Research Trust to Dr Place. Dr Tang was supported by a grant from the Nancy Lurie Marks Family Foundation awarded to Prof Skuse. Author contributions: Prof Skuse conceptualised the research and conducted, along with other clinicians, the clinical interviews. Prof Skuse, Dr Mandy and Dr Tang contributed to data analysis, interpretation of findings and preparation of the manuscript. Competing interests: The authors declare that they have no competing interests, financial or otherwise, in completion of this work. Data and materials availability: All data and materials are available are upon request to the corresponding author. 